\title{
14.
}

\section{Sulle equationi lineari alle differenze finite.}

(Nola di P. Turdy, membro corrispondente dell'Accademia Pontifica de'Nuovi Lincei.)

(Estratta dagli Annali di Scienze Mathematiche e Fisiche pubblicati in Roma. Agosto 1850.)

Sia data un'equazione lineare alle differenze finite dell'ordine $n$,

(1.) $\boldsymbol{y}_{x+n}+\boldsymbol{P}_{1} y_{x+n-1}+\boldsymbol{P}_{2} \boldsymbol{y}_{x+n-2}+\cdots+\boldsymbol{P}_{n} \boldsymbol{y}_{x}=\mathbf{0}$,

ed un'altra pur lineare dell'ordire $m>n$ che debba coesistere con la prima, che abbia ciò̀ comuni con essa tutti gl'integrali particolari

(2.) $\boldsymbol{z}_{x+m}+\boldsymbol{A}^{(1)} \boldsymbol{z}_{x+m-1}+\boldsymbol{A}^{(2)} \boldsymbol{z}_{x+m-2}+\cdots+\boldsymbol{A}^{(m)} \boldsymbol{z}_{x}=0$.

II valore generale di $z$ conterrà $m$ constanti, quello di $y$ ne conterrà $n$ e pero sarà della forma

$$
y=z=C_{1} y^{(1)}+C_{2} y^{(2)}+\cdots+C_{n-m} y^{(n-m)},
$$

ove $y^{(1)}, y^{(2)}, \ldots y^{(n-m)}$ rappresentano gli $n-m$ integrali particolari esclusivi della (1.).

\section{Facciamo}

(3.) $\quad \boldsymbol{u}_{x}=\boldsymbol{y}_{x+m}+\boldsymbol{A}^{(1)} \boldsymbol{y}_{x+m-1}+\boldsymbol{A}^{(2)} \boldsymbol{y}_{x+m-2}+\cdots+\boldsymbol{A}^{(m)} \boldsymbol{y}_{x}$,

e sostituendo per $y$ il suo valore $z$ sparirà da sè in virtù delle (2.), ed $u_{x}$ risulterà una funzione della $x$ contenente $n-m$ costanti arbitrarie in modo lineare. Però eliminando queste si avrà un'equazione lineare alle differenze finite dell' ordine $n-m$ che dinoteremo con

$$
\boldsymbol{u}_{x+n-m}+\boldsymbol{a}_{1} \boldsymbol{u}_{x+n-m-1}+\boldsymbol{a}_{2} \boldsymbol{u}_{x+n-m-2}+\cdots+\boldsymbol{a}_{n-m} \boldsymbol{u}_{x}=\mathbf{0} .
$$

Rimeltendo in questa per $u_{x}$ il suo valore (3.) e paragonando la risultante con la proposta (1.), otterremo $n$ equazioni

$$
\begin{aligned}
& \boldsymbol{A}_{x+n-m}^{(1)}+\boldsymbol{a}_{1}=\boldsymbol{P}_{1}, \quad A_{x+n-m}^{(2)}+\boldsymbol{a}_{1} \boldsymbol{A}_{x+n-m-1}^{(1)}+\boldsymbol{a}_{2}=\boldsymbol{P}_{2}, \\
& \boldsymbol{A}_{x+n-m}^{(3)}+\boldsymbol{a}_{2} A_{x+n-m-1}^{(2)}+\boldsymbol{a}_{2} \boldsymbol{A}_{x+n-m-2}^{(1)}+\boldsymbol{a}_{3}=\boldsymbol{P}_{3}, \text { ec. }
\end{aligned}
$$

che procedono con legge manifesta e delle quali le prime $n-m$ serviranno a determinare i valori di $a_{1}, a_{2}, \ldots a_{n-m}$, e le altre ne forniranno le condizioni cui debbono verificare i coefficienti della (1.) e della (2.) perchè esse coesistano. Cosi la soluzione della (1.) è ricondotta a quella di due equa- 
zioni: una, la (3.) dell' ordine $m$, e la seconda, la (4.), dell' ordine $n-m$, ed i coefficienti di quest'ultima si ottengono senza alcuna integrazione. Questo è il teorema fondamentale del sig. Libri trasportato alle equazioni alle differenze finite, e la dimostrazione qui esposta è perfettamente analoga a quella data dal sig. Liouville per le equazioni differenziali.

Sieno ora dati $n-1$ integrali particolari della (1.); egli è noto che l'integrazione di essa si riduce a quella di un'equazione lineare del $1^{\circ}$. ordine, e che quindi si può trovare l'altro integrale particolare per formare il completo; ma se co'metodi ordinari si volesse questo assegnare, sarebbe pressochê impossibile scriverne la espressione finale. Però se introduciamo l'uso delle funzioni alternate o determinanti riescirà agevole condurre il calcolo sino in fondo.

Indichino $y^{(1)}, y^{(2)}, \ldots y^{(n-1)}$ gli $n-1$ integrali particolari dati, e rappresenti

$$
\mathfrak{z}_{x+n-1}+\boldsymbol{A}^{(1)} \boldsymbol{z}_{x+n-2}+\boldsymbol{A}^{(2)} \mathfrak{z}_{x+n-3}+\cdots+\boldsymbol{A}^{(n-1)} \boldsymbol{z}_{x}=\mathbf{0}
$$

l'equazione dell'ordine $n-1$ cui essi appartengono. Avremo per determinare i coefficienti $A^{(1)}, A^{(2)}, \ldots A^{(n-1)}$ le $n-1$ seguenti equazioni

$$
\begin{gathered}
y_{x+n-1}^{(1)}+A^{(1)} y_{x+n-2}^{(1)}+\cdots+A^{(n-1)} y_{x}^{(1)}=0 \\
y_{x+n-1}^{(1)}+A^{(1)} y_{x+n-2}^{(2)}+\cdots+A^{(n-1)} y_{x}^{(2)}=0, \\
\cdot \cdot \cdot \cdot \cdot \cdot \cdot \cdot \cdot \cdot \cdot \cdot \cdot \cdot \cdot \cdot \cdot \cdot \cdot \cdot \cdot \cdot \cdot \cdot \dot{A}^{(n-1)} y_{x}^{(n-1)}=0 .
\end{gathered}
$$

Il solo che importa conoscere $A^{(1)}$ è somministrato dalla formula

$$
\boldsymbol{A}^{(1)}=\frac{-\boldsymbol{S}\left\{ \pm y_{x}^{(1)} \cdot y_{x+1}^{(2)} \ldots y_{x+n-3}^{(n-2)} \cdot y_{x+n-1}^{(n-1)}\right\}}{S\left\{ \pm y_{x}^{(1)} \cdot y_{x+1}^{(2)} \ldots y_{x+n-3}^{(n-2)} \cdot y_{x+n-2}^{(n-1)}\right\}}
$$

(Indico con $\boldsymbol{S}$ il determinante anzichè con $\Sigma$ perchè quest' ultimo simbolo è qui impiegato nel senso ordinario d'integrazione finita.)

Ciò posto la. (1.) e la (5.) avendo a comune $n-1$ integrali particolari, siamo nel caso del teorema precedente con $m=n-1$, e però la soluzione cercata dipenderà da quello delle due equazioni

$$
\begin{gathered}
\boldsymbol{u}_{x+1}+\left\{\boldsymbol{P}_{1}-\boldsymbol{A}_{x+1}^{(1)}\right\} \boldsymbol{u}_{x}=\mathbf{0} \\
\boldsymbol{y}_{x+n-1}+\boldsymbol{A}^{(\mathbf{1})} y_{x+n-2}+\cdots+\boldsymbol{A}^{(n-1)} y_{x}=u_{x} .
\end{gathered}
$$

Dalla prima si ricava

$$
u_{x}=C_{n} e^{\Sigma \log \left\{A_{x+1}^{(1)}-P_{1}\right\}}
$$

Crelle's Journal f. d. M. Bd. XLII. Heft 2. 
e dinotando il numeratore $\mathrm{di} \boldsymbol{A}^{(1)}$ con $\boldsymbol{N}$ ed il denominatore con $\boldsymbol{D}$, allorquando la $x$ diviene $x+1$, sarà

$$
u_{x}=C_{n} e^{\Sigma \log \left\{\frac{N}{D}-P_{1}\right\}}
$$

L'integrale della seconda si otterrà per mezzo della variazione delle costanti arbitrarie conoscendo già tutti gl'integrali particolari di essa quando il secondo membro è nullo: poniamo quindi

e mettiamo

$$
y_{x}=y^{(1)} z_{1}+y^{(2)} z_{2}+\cdots+y^{(n-1)} z_{n-1}
$$

$$
\Delta \mathfrak{z}_{1}=v_{1} u, \ldots \Delta \mathfrak{z}_{r}=v_{r} u, \ldots \Delta z_{n-1}=v_{n-1} u,
$$

le funzioni $v_{1}, \ldots v_{1}, \ldots v_{n-1}$ saranno determinate dalle equazioni

$$
\begin{aligned}
& y_{x-1}^{(1)} v_{1}+y_{x+1}^{(2)} v_{2}+\cdots+y_{x+1}^{(r)} v_{r}+\cdots+y_{x+1}^{(n-1)} v_{n-1}=0, \\
& y_{x+2}^{(1)} v_{1}+\cdots+y_{x+2}^{(r)} v_{r}+\cdots+y_{x+2}^{(n-1)} v_{n-1}=0 \text {, } \\
& y_{x+n-1}^{(1)} v_{1}+\cdots+y_{x+n-1}^{(r)} v_{r}+\cdots+y_{x+n-1}^{(n-1)} v_{n-1}=1 .
\end{aligned}
$$

Il valore generico di $\boldsymbol{v}_{r}$ sarà

$$
\boldsymbol{v}_{r}=\frac{\boldsymbol{S}\left\{ \pm y_{x+1}^{(1)} \cdots \cdot y_{x+r-1}^{(r-1)} \cdot y_{x+r}^{(n-1)} \cdot y_{x+r-1}^{(r+1)} \cdot y_{x+n-2}^{(n-2)}\right\}}{\boldsymbol{S}\left\{ \pm y_{x+1}^{(1)} \cdots y_{x+r-1}^{(r-1)} \cdot y_{x+r}^{(n-1)} \cdot y_{x+r+1}^{(r+1)} \cdot y_{x+n-2}^{(n-2)} \cdot y_{x+n-1}^{(r)}\right\}}=\frac{1}{\boldsymbol{D}} \cdot \frac{d \boldsymbol{D}}{d y_{x+n-1}^{(r)}}
$$

e verrà

$$
z_{r}=C_{n} \Sigma \frac{1}{D} \cdot \frac{d D}{d y_{x+n-1}^{(r)}} e^{\Sigma\left\{\frac{N}{D}-P_{1}\right\}}+C_{r}
$$

Questo teorema corrisponde a quello enunciato dal professore Malmstèn per le equazioni differenziali nel No. 21 del Dublin and Cambridge Mathematical Iournal, e la dimostrazione è perfettamente analoga a quella da me data di quest' ultimo nel fascicolo di Aprile di questi Annali.

Esempio I. $n=2$, la equazione data sarà

$$
\boldsymbol{y}_{x+2}+\boldsymbol{P}_{1} y_{x+1}+\boldsymbol{P}_{2} y_{x}=0
$$

e l'integrale particolare noto $y^{(1)}$. Dalla nostra formola risulta per l'integrale completo

$$
y_{x}=y^{(1)} z_{1}=C_{1} y^{(1)}+C_{2} y^{(1)} \Sigma \frac{1}{y_{x+1}^{(1)}} e^{\Sigma \log \left\{-\frac{y_{x+2}^{(1)}}{y_{x+1}^{(1)}}-P_{1}\right\}} .
$$

E facile ricavare il secondo integrale particolare facendo al solito $y_{x}=y^{(1)} \Sigma t_{x}$. 
14. Tardy, sulle equazioni lineari alle differenze finite.

Esempio II. Per $n=3$, cioè per la equazione

$$
y_{x+3}+\boldsymbol{P}_{1} y_{x+2}+\boldsymbol{P}_{2} y_{x+1}+\boldsymbol{P}_{3} y_{x}=0 \text {. }
$$

Supposti noti $y^{(1)}$ ed $y^{(2)}$ risulta

$$
\begin{gathered}
y_{2}=C_{1} y^{(1)}+C_{2} y^{(2)} \\
+C_{3}\left\{y^{(1)} \Sigma \frac{y_{x+1}^{(2)}}{y_{x+1}^{(1)} y_{x+2}^{(2)}-y_{x+2}^{(1)} y_{x+1}^{(2)}} e^{\sum \log \left\{\frac{y_{x+3}^{(1)} y_{x+1}^{(2)}-y_{x+1}^{(1)} y_{x+3}^{(2)}}{y_{x+1}^{(1)} y_{x+2}^{(2)}-y_{x+2}^{(1)} y_{x+1}^{(2)}}-P_{1}\right\}}\right. \\
\left.-y^{(2)} \Sigma \frac{y_{x+1}^{(1)}}{y_{x+1}^{(1)} y_{x+2}^{(2)}-y_{x+2}^{(1)} y_{x+1}^{(2)}} e^{\sum \log \left\{\frac{y_{x+3}^{(1)} y_{x+1}^{(2)}-y_{x+1}^{(1)} y_{x+3}^{(2)}}{y_{x+1}^{(1)} y_{x+2}^{(2)}-y_{x+2}^{(1)} y_{x+1}^{(2)}-P_{1}}\right\}}\right\} .
\end{gathered}
$$

Riesce assai spedito vedere come questa espressione, alla quale non è cosi semplice ridurre quella che si otterrebbe al modo ordinario, verifica la proposta.

Firenze 3 luglio 1850. 